\title{
Polyhedral hyperbolic metrics on surfaces
}

\author{
François Fillastre
}

Published online: 15 November 2008

(C) Springer Science+Business Media B.V. 2008

\section{Erratum to: Geom Dedicata DOI: 10.1007/s10711-008-9252-2}

The original version of the article presented some ambiguity due to omitted aspects. The text below serves to clarify.

In the last section of [4] it is proved that the map $\mathcal{I}$ is a finite-sheeted covering map between $\mathcal{P}$ and $\mathcal{M}$. As $\mathcal{M}$ is simply connected it is deduced that $\mathcal{I}$ is a homeomorphism. The fact that $\mathcal{P}$ is connected is missing. Here, we provide a proof which is a simple adaptation of the argument for the case of the sphere [1, 9.1.2]. The Fuchsian case with finite vertices was already done in [3].

For the parabolic case it is convenient to introduce a projective model of the hyperbolicde Sitter space, which is a direct extension of a model of the hyperbolic space known as paraboloid model [5, 2.3.13] or as parabolic model [2, 10.25]. Let $\ell$ be a light-like vector of $\mathbb{R}_{1}^{4}$, the Minkowski space of dimension 4 . The paraboloid model can be obtained by a central projection in $\mathbb{R}_{1}^{4}$ of $\widetilde{\mathrm{HS}}_{\ell}^{3}$ onto a light-like hyperplane parallel to $\ell^{\perp}$. In this model $\widetilde{\mathrm{HS}}_{\ell}^{3}$ is homeomorphic to $\mathbb{R}^{3}$, geodesics are sent to straight lines, the boundary $\ell^{\perp}$ of $\mathrm{dS}_{\ell}^{3}$ is sent to infinity and we choose coordinates such that $\ell$ is sent to $(0,0, \infty)$. We denote by $D$ the part of the boundary at infinity of $\mathbb{H}^{3}$ sent to the paraboloid of equation $2 z=-x^{2}-y^{2}$. The hyperbolic space is sent below $D$ and $\mathrm{dS}_{\ell}^{3}$ is sent above $D$. Horospheres centered at $\ell$ are sent to paraboloids $2 z=-x^{2}-y^{2}+t$, where $t$ is a real number. The orthogonal projection onto the horospheres centered at $\ell$ along the lines starting from $\ell$ corresponds to the vertical projection in this model.

The online version of the original article can be found under doi:10.1007/s10711-008-9252-2.

F. Fillastre $(\varangle)$

Département de Mathématiques, Université de Cergy-Pontoise/Saint-Martin, 2, avenue Adolphe Chauvin, 95302 CERGY-PONTOISE Cedex, France e-mail: francois.fillastre@u-cergy.fr URL: http://www.u-cergy.fr/ffillast/ 
For the Fuchsian case we consider the Klein projective model, the plane fixed by the Fuchsian groups is the horizontal one and we take for $D$ the upper half part of the unit sphere. The orthogonal projection onto caps corresponds to the vertical projection in this model.

Let $(P, G) \in \mathcal{P}$. We denote by $\mathcal{P}^{(P, G)}$ the subset of $\mathcal{P}$ made with elements $(Q, G)$ such that $Q$ has its vertices on the same vertical lines as the vertices of $P$. We also denote by $\overline{\mathcal{P}}^{(P, G)}$ the corresponding subset in the space of invariant convex HS-polyhedra (i.e. we remove the condition that edges must meet hyperbolic space).

The height of a vertex $v$ is the signed vertical Euclidean distance between $D$ and $v$. For an ideal vertex the height is zero. It is positive for a strictly hyperideal vertex and negative for a finite vertex. For example in the parabolic case if $v$ has coordinates $(a, b, c)$, its height is $c+\left(a^{2}+b^{2}\right) / 2$. Each element of $\overline{\mathcal{P}}^{(P, G)}$ is defined by the $(n+m+p)$ heights of vertices which are inside a fundamental domain for the action of $G$ [the polyhedral surface is then the convex hull of the orbits for $G$ of these $(n+m+p)$ vertices]. In the following we identify $\overline{\mathcal{P}}^{(P, G)}$ with a subset of $\mathbb{R}^{n+m+p}$.

The condition for an element of $\mathbb{R}^{n+m+p}$ to correspond to an element of $\overline{\mathcal{P}}^{(P, G)}$ is that each vertex must lie outside the convex hull of the other vertices. That means that if $v$ is one of the $(n+m+p)$ vertices and $v_{1}, v_{2}, v_{3}$ are any other vertices such that $v$ is contained inside the vertical prism defined by $v_{1}, v_{2}, v_{3}$, then the plane spanned by $v_{1}, v_{2}, v_{3}$ must lie below $v$. This gives (an infinite number of) affine conditions on the heights of the $(n+m+p)$ vertices. We then get a convex subset of $\mathbb{R}^{n+m+p}$ and if $\left(x_{1}, \ldots, x_{n+m+p}\right)$ are the coordinates of the Euclidean space, we have to intersect this convex subset with $x_{i}<0$ if the $i$ th coordinate corresponds to a finite vertex, with $x_{i}>0$ if it corresponds to a strictly hyperideal vertex and with $x_{i}=0$ if it is a finite vertex. Hence, $\overline{\mathcal{P}}^{(P, G)}$ is a convex subset of $\mathbb{R}^{n+m}$.

Let us call $\left(P^{i}, G\right)$ the ideal convex invariant polyhedron whose vertices are the vertical projection of the ones of $P$ onto $D\left[\left(P^{i}, G\right)\right.$ is the origin of $\mathbb{R}^{n+m}$ for the coordinates we introduced]. It is lying on the boundary of $\overline{\mathcal{P}}^{(P, G)}$ as slightly pushing up and down suitable vertices of $\left(P^{i}, G\right)$ gives an element of $\overline{\mathcal{P}}^{(P, G)}$.

Hence in $\overline{\mathcal{P}}^{(P, G)}$ there is a segment $s$ from $(P, G)$ to 0 . Actually $s$ is lying in $\mathcal{P}^{(P, G)}$ : along $s$ the heights of the strictly hyperideal vertices decrease, and if we start from a polyhedron with all edges meeting hyperbolic space, this property is preserved all along the deformation.

Let us denote by $\mathcal{P}^{i}$ the set of ideal convex invariant polyhedra with $(n+m+p)$ vertices in a fundamental domain. We described a continuous path in $\mathcal{P}$ from any element of $\mathcal{P}$ such that the other endpoint of its completion is in $\mathcal{P}^{i}$. This last space is path-connected as it is homeomorphic to the Teichmüller space of $(n+m+p)$ marked points on the compact surface $\bar{S}$. Moreover, any continuous path in $\mathcal{P}^{i}$ can be approximated by a path in $\mathcal{P}$. Hence, $\mathcal{P}$ is path connected.

\section{References}

1. Alexandrov, A.D.: Convex Polyhedra. Springer Monographs in Mathematics. Springer-Verlag, Berlin (2005)

2. Bridson, M.R., Haefliger, A.: Metric Spaces of Non-positive Curvature. Volume 319 of Grundlehren der Mathematischen Wissenschaften [Fundamental Principles of Mathematical Sciences]. Springer-Verlag, Berlin (1999)

3. Fillastre, F.: Polyhedral realisation of hyperbolic metrics with conical singularities on compact surfaces. Ann. Inst. Fourier (Grenoble) 57(1), 163-195 (2007)

4. Fillastre, F.: Polyhedral hyperbolic metrics on surfaces. Geom. Dedicata 134, 177-196 (2008)

5. Thurston, W.P.: Three-dimensional geometry and topology. In: Levy, S. (ed.) Volume 35 of Princeton Mathematical Series, vol. 1. Princeton University Press, Princeton, NJ (1997) 